Short Research Communication

\title{
All Human EFI $\alpha$ Promoters Are Not Equal: Markedly Affect Gene Expression in Constructs from Different Sources
}

\author{
Changyu Zheng ${ }^{\bowtie}$ and Bruce J. Baum \\ Molecular Physiology and Therapeutics Branch, National Institute of Dental and Craniofacial Research, NIH, DHHS, Bethesda, MD \\ 20892-1190. \\ $\triangle$ Corresponding author: Dr. Changyu Zheng, MPTB/NIDCR/NIH, Bldg. 10, Room 1A01, MSC-1190, 9000 Rockville Pike, Bethesda, MD \\ 20892-1190. Tel: (301)594-1924 Fax: (301)402-1228 Email: czheng@mail.nih.gov. \\ (0) Ivyspring International Publisher. This is an open-access article distributed under the terms of the Creative Commons License (http://creativecommons.org/ \\ licenses/by-nc-nd/3.0/). Reproduction is permitted for personal, noncommercial use, provided that the article is in whole, unmodified, and properly cited.
}

Received: 2013.II.0I; Accepted: 2014.0I.09; Published: 2014.03.07

\begin{abstract}
The promoter is a major element in the expression cassette of gene therapy vectors. Optimal promoter selection can enhance target specificity and gene expression. Recently, we evaluated three different human elongation factor I alpha (EFI $\alpha$ ) promoters. The three promoters were put into the same expression vector, pAC-luc, driving expression of the luciferase cDNA. The activity from one EFI a promoter (termed EFI $\alpha-3$ ), obtained in a commercial vector, was markedly lower when tested in vitro (from $50-500 \mathrm{x}$ ) in four cell lines and in vivo in rat submandibular glands $(\sim 250 \mathrm{x})$. Sequence differences in the EFI $\alpha-3$ promoter likely account for the activity differences seen. Investigators need to recognize that all promoters of the same name may not be equivalent in driving transgene expression.
\end{abstract}

Key words: promoter, EF1a, gene therapy.

\section{Introduction}

The expression cassette is an essential component of a gene therapy vector, and each includes at least three key components: a promoter, a transgene and a polyadenylation sequence. The optimal design of an expression cassette can facilitate therapeutic outcomes $(1,2,3)$. The promoter plays a critical role, including directing, regulating and targeting transgene expression. Higher promoter activity can result in lower doses of vector administered, with consequently lower toxicity or side effects.

Currently, many promoters are available for the gene therapy studies. Differences, however, are known to exist within promoters of the same name. For example, the CMV promoter (including human cytomegalovirus enhancer and immediate early promoter) used in different vectors was $589 \mathrm{bp}$ in size in Ad-EGFP (4), $646 \mathrm{bp}$ in pCNS (5), $742 \mathrm{bp}$ in phMGFP (accession No. in NCBI: AY218848), 795 bp in pCMVTNT(TM)(accession No. in NCBI: AF477200), 817 bp in expression vector M64754 (accession No. in
NCBI: M64754) and $1650 \mathrm{bp}$ in expression vector AF286076 (accession No. in NCBI: AF286076). All are simply called the CMV promoter. It is not known whether such sequence differences result in significant functional differences.

Recently, in an effort to optimize expression cassettes for planned human trials, we have studied three versions of the human EF1a promoter, two obtained from commercial vectors and one from a colleague. Although, all are called the EF1a promoter, the functional activity from one commercially-obtained promoter was markedly lower in four different cell lines in vitro and in vivo in rat submandibular glands.

\section{Materials and Methods}

\section{Construction of plasmids}

A backbone plasmid, pAC-luc (6), was constructed using pACCMV-pLpA that was a gift from 
Dr. C. Newgard (University of Texas, Southwestern Medical Center). This plasmid contains part of the adenovirus serotype 5 genome (0-1.2 and 9.2-17 $\mathrm{mu}$ ) with the human CMV immediate early promoter/enhancer substituted between map units 1.3 and 9.1. pACCMV-pLpA was digested with Not I to delete the CMV promoter/enhancer and SV40 poly A sequence. A 307 bp DNA fragment, containing a multiple cloning site (Bgl II, Xho I, Hind III, EcoR I, Sal I, Kpn $\mathrm{I}, \mathrm{Bam \textrm {H } I}$ and $\mathrm{Xba} \mathrm{I}$ ) and SV40 poly A sequence, was removed from pEGFP-C3 (BD Biosciences, Palo Alto, CA) with Mlu I and Sca I, and filled in with Klenow large DNA fragment (Invitrogen, Carlsbad, CA). Not I linkers were added and then ligated with the remaining pACCMV-pLpA fragment to become the pAC plasmid. Next, a 1851 bp luciferase cDNA (Kpn I/ BamH I fragment from pGL2-Basic, Promega, Madison, WI) was inserted into the pAC Kpn I/BamH I sites to generate $\mathrm{pAC}$-luc. The first EF1 $\alpha$ promoter used (1197 bp), termed EF1a-1 in this study, was excised from the plasmid pEAK8 (Edge BioSystems, Gaithersburg, MD) with Spe I, filled in with Klenow large DNA fragment, and then digested with Eco RI. Next, pAC-luc was digested with Hind III, filled in with Klenow large DNA fragment, then digested with Eco RI and ligated with EF1a-1. The second EF1 $\alpha$ promoter, termed EF1a-2 (1272 bp), was a gift from Dr. S. Gutkind (OPCB, NIDCR, NIH) (7), and was excised from pEF-BOS with EcoR I/Hind III and inserted into pAC-luc. The last EF1 $\alpha$ promoter studied here, termed EF1a-3 (1191 bp), was excised from $\mathrm{pEF1/myc/ER/GFP} \mathrm{(Invitrogen)} \mathrm{with} \mathrm{Eco} \mathrm{RI/Nco} \mathrm{I,}$ filled in with Klenow large DNA fragment, and then Hind III linkers were added to both ends. EF1a-3 was inserted into pAC-luc at site the Hind III site.

\section{Cell culture}

The A5 cell line was derived from rat submandibular gland (8) and grown in McCoy's 5A medium (Invitrogen). The HSY cell line was obtained from a human parotid adenocarcinoma (9) and grown in a mixture of $50 \%$ Dulbecco's minimum essential medium (DMEM) and 50\% Ham's F12 media. The HSG cell line was obtained from an irradiated human submandibular gland (10) and grown in DMEM/F12 medium, as above. The 293 cell line (Microbix Biosystems Inc., Toronto, Ontario, Canada) is from human embryonic kidney (11) and was grown in Eagle's minimum essential medium. For all four cell lines the following supplements were included: $10 \%$ fetal bovine serum (Invitrogen,), $100 \mathrm{U} / \mathrm{ml}$ penicillin G (Invitrogen), $100 \mu \mathrm{g} / \mathrm{ml}$ streptomycin (Invitrogen). Cells were incubated at $37^{\circ} \mathrm{C}$ in a humidified $5 \% \mathrm{CO}_{2}$ atmosphere.

\section{In vitro and in vivo plasmid transfection}

We used adenovirus- polyethylenimine (PEI)-plasmid complexes to deliver plasmids into A5, HSG, HSY and 293 cells in vitro and rat submandibular gland in vivo. PEI (Sigma, St. Louis, MO) has a high cationic-charge-density potential and is useful to enhance transfection $(12,13)$. The adenovirus used for all transfections herein was Adcontrol, an E1- recombinant adenovirus without any transgene. Adcontrol was generated by cotransfection of pACCMV-pLpA and pJM17 in C7 cells (6). The complexes were formed using $2 \times 10^{10}$ molecules of plasmid DNA/ $4 \times 10^{5}$ cells, $0.1 \mathrm{mM}$ of PEI and $3 \times 10^{10}$ particles of Adcontrol in a volume of $20 \mu \mathrm{l}$. A5, HSG, HSY and 293 cells were plated at $2 \times 10^{5}$ cells/well in 96 well plates. After 24 hours, the cells were transfected with $20 \mu \mathrm{l}$ of adenovirus-PEI-plasmid complexes for 1 hour, then $180 \mu \mathrm{l}$ of fresh growth medium was added to each well. Cells were cultured for an additional 24 hours and then luciferase activity was measured.

Male Wistar rats (250-350 g, 3 months old) were anesthetized $(14,15,16)$ and the adenovirus-PEI-plasmid complexes for plasmid transfection, which were formed using $4.35 \times 10^{12}$ molecules of plasmid DNA/gland, $0.5 \mathrm{mM}$ of PEI and $1 \times 10^{11}$ particles of Adcontrol in a volume of $200 \mu \mathrm{l}$, were administered by retrograde ductal instillation $(14,15,16)$ into both submandibular glands. Three rats were studied for each experimental group. After 3 days, rats were euthanized and submandibular glands collected for analysis of luciferase activity. All animal studies were approved by the NIDCR Animal Care and Use Committee.

\section{Luciferase assays}

A5, HSG, HSY and 293 cells were lysed 24 hours post-transfection with cell lysis buffer (Promega) for $15 \mathrm{~min}$ at room temperature. For rat submandibular tissues, about $100 \mathrm{mg}$ wet weight of samples were added to $500 \mu \mathrm{l}$ of $1 \times$ cell lysis buffer (Promega). Samples were homogenized with a homogenizer from OMNI international (Waterbury, CT) and incubated for $15 \mathrm{~min}$ at room temperature. Fifty microliters of the cell lysates were transferred to assay tubes, $100 \mu \mathrm{l}$ of luciferase substrate injected, and light output was measured with an OPTOCOMP I luminometer (GEM Instruments, INC., Hamden, CT) for a $10 \mathrm{sec}$ interval. After the measurement of luciferase activity, the concentration of protein in separate aliquots of the lysate was measured using the BCA protein assay kit (PIERCE, Rockford, IL). Results were expressed as relative light unit (RLU)/ mg protein.

\section{Data analysis}

Data analyses employed SigmaStat version 2.0 
(SPSS Inc, Chicago, IL). Descriptive statistics were calculated and are reported as mean $\pm \mathrm{SD}$. One-way ANOVAs, following by a Tukey test, were used to determine the statistical significance of differences. Values of $p<0.05$ were considered significant.

\section{Results and Discussion}

\section{EF I $\alpha$ promoter activity}

On average, the EF1 $\alpha-1$ and EF1 $\alpha-2$ promoters showed the highest activities in all cell lines. The EF1 $\alpha-1$ promoter activity was statistically higher in A5 and HSG cells ( $2 \mathrm{x} ; \mathrm{p}<0.001)$, but there were no statistical differences between the activity of these promoters in HSY and 293 cells (Figure 1). However, as seen in Figure 1, it is clear that luciferase activity with the EF1 $\alpha-3$ promoter was significantly lower than those seen with the EF1 $\alpha-1$ and EF1 $\alpha-2$ promoters in all four cell lines $(\mathrm{p}<0.001) ; \sim 50$-fold lower in A5 cells, $\sim 145$-fold lower in HSG cells, $\sim 80$-fold lower in HSY cells and $\sim 500$-fold lower in 293 cells.
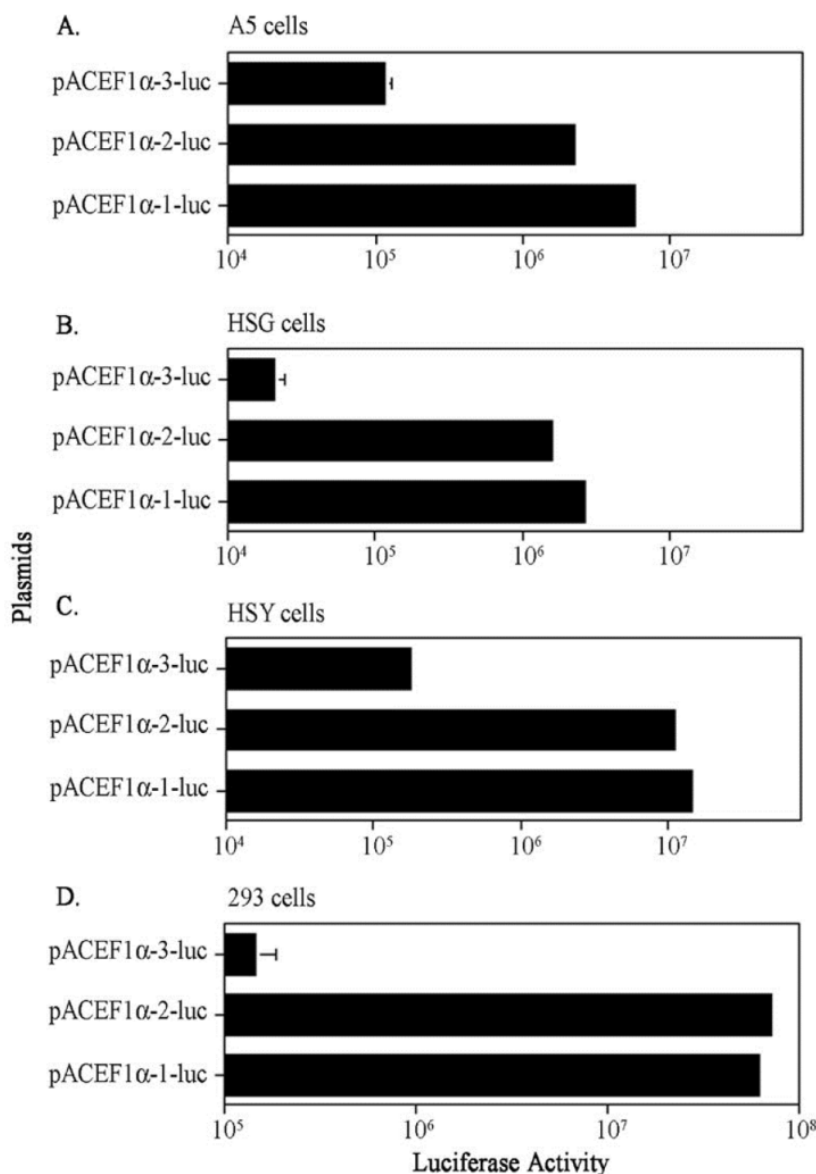

Figure I. Comparison of three EFI $\alpha$ promoter activities in vitro after transfection using plasmid-PEl-Adcontrol complexes. A. Promoter activity in A5 cells; B. Promoter activity in HSG cells; C. Promoter activity in HSY cells; D. Promoter activity in 293 cells. Each cell was transfected with $5 \times 10^{4}$ plasmid molecules. See text for details. The data shown are the means \pm SD of luciferase activity from three determinations $24 \mathrm{hr}$ after transfections and are representative of three separate experiments. For most determinations the error bars are too small to be visualized in the figure.
To determine if there was a similar difference in the activity of the different EF1 $\alpha$ promoters in vivo, we tested two plasmids, pAC EF1 $\alpha-1$-luc and pAC EF1 $\alpha$-3-luc, in rat submandibular glands. The plasmids were transfected at $4.35 \times 10^{12}$ molecules/gland using plasmid-PEI-Adcontrol complexes, and luciferase activity was measured at 3 days post-transfection (Figure 2). As the data in Figure 2 show, the luciferase activity observed in vivo with the EF1 $\alpha-3$ promoter was also markedly lower ( 250 -fold) than that with the EF1 $\alpha-1$ promoter $(p<0.01)$.

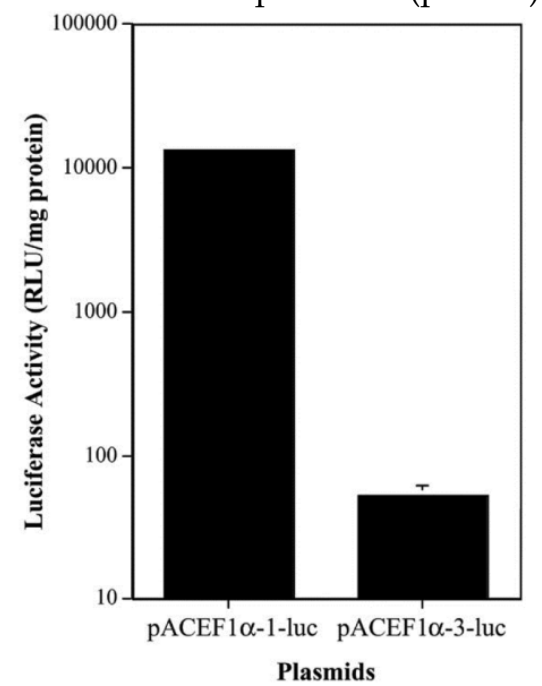

Figure 2. Comparison of two EFI $\alpha$ promoter activities in vivo in rat submandibular glands after transfection using plasmid-PEI-Adcontrol complexes. Each submandibular gland was transfected with $4.35 \times 10^{12}$ plasmid molecules. See text for details. The data shown are the means \pm SD of luciferase activity from six submandibular glands of three rats measured three days after transfections. Note that for the pAC EFI $\alpha$-I-luc data the error bars are too small to be visualized in the figure.

\section{EF I $\alpha$ promoter sequence analysis}

To better appreciate possible reasons for the significantly different expression seen from the EF1 $\alpha-3$ promoter, we sequenced all three EF1 $\alpha$ promoters in their entirety. We also searched a human EF1 $\alpha$ gene in GenBank (NCBI website, accession number: J04617)(17) and used this sequence to compare with the sequences obtained from the EF1 $\alpha-1$, EF $1 \alpha-2$ and EF1 $\alpha-3$ promoters studied here. Differences between the J04617 sequence and the EF1 $\alpha-1$, $E F 1 \alpha-2$ or EF1 $\alpha-3$ promoters are shown in Figure 3. The EF1 $\alpha-1$ promoter (panel A) started at nt 374 in J04617. There were two differences between these sequences. One was from nt 685 to 688 in the J04617, i.e. these four base pairs (CGCC) were missing in EF1 $\alpha-1$. The other was located between nt 948 and 949 in J04617; EF1 $\alpha-1$ had three extra base pairs, GAC, inserted. Panel B shows that the EF1 $\alpha-2$ promoter exhibited two differences from the J04617 sequence in GenBank. First, in EF1 $\alpha-2$ there were 81 extra base 
pairs upstream of J04617 promoter sequence. After searching in GenBank, these 81 base pairs were identified as being derived from the SV40 small antigen. Second, as in EF1 $\alpha-1$, four base pairs (CGCC) were absent in EF1 $\alpha-2$ from nt 685 to 688 in J04617. Panel C shows the comparison between J04617 and the EF1 $\alpha-3$ promoter, and shows three types of differences between them. First, there were thirteen point mutations found in the EF1 $\alpha-3$ promoter, spread throughout the sequence. Second, the same four base pairs (CGCC; nt 685-688) missing in EF1 $\alpha-1$ and EF1 $\alpha-2$ also were absent in the EF1 $\alpha-3$ promoter. Third, the sequence in EF1 $\alpha-3$ following nt 357 showed multiple differences from the comparable region of J04617 (from nt 721 to 751). Is there any transcription factor binding site in these 30 base pairs? Two programs were used to search the binding sites. Seventeen potential transcription factor binding sites, p300, Egr-3, EIIaE-A, R2, Elk-1, VDR, WT1 I, STAT5A, c-Myb, HMG I(Y), STAT4, c-Ets-1, NFI/CIF, Ik-1, MAZ, HSF1 (long) and HSF1 (short) were found by PROMO $(18,19)$. Using TFBIND (20) program, we found 23 potential transcription factor binding sites, SP1, c-Myb, Elk1, RREB1, p300, AP2, NGFIC, EGR1, EGR2, EGR3, MZF1, ZID, LYF1, AHRARNT, AP4, NFKB, NFKAPPAB50, HSF1, HSF2, ARP1, E2F, STAF and PAX5. These indicate that these 30 base pairs could play important roles in the regulation of EF1 $\alpha$ promoter activity.

\section{EF $1 \alpha-3$}

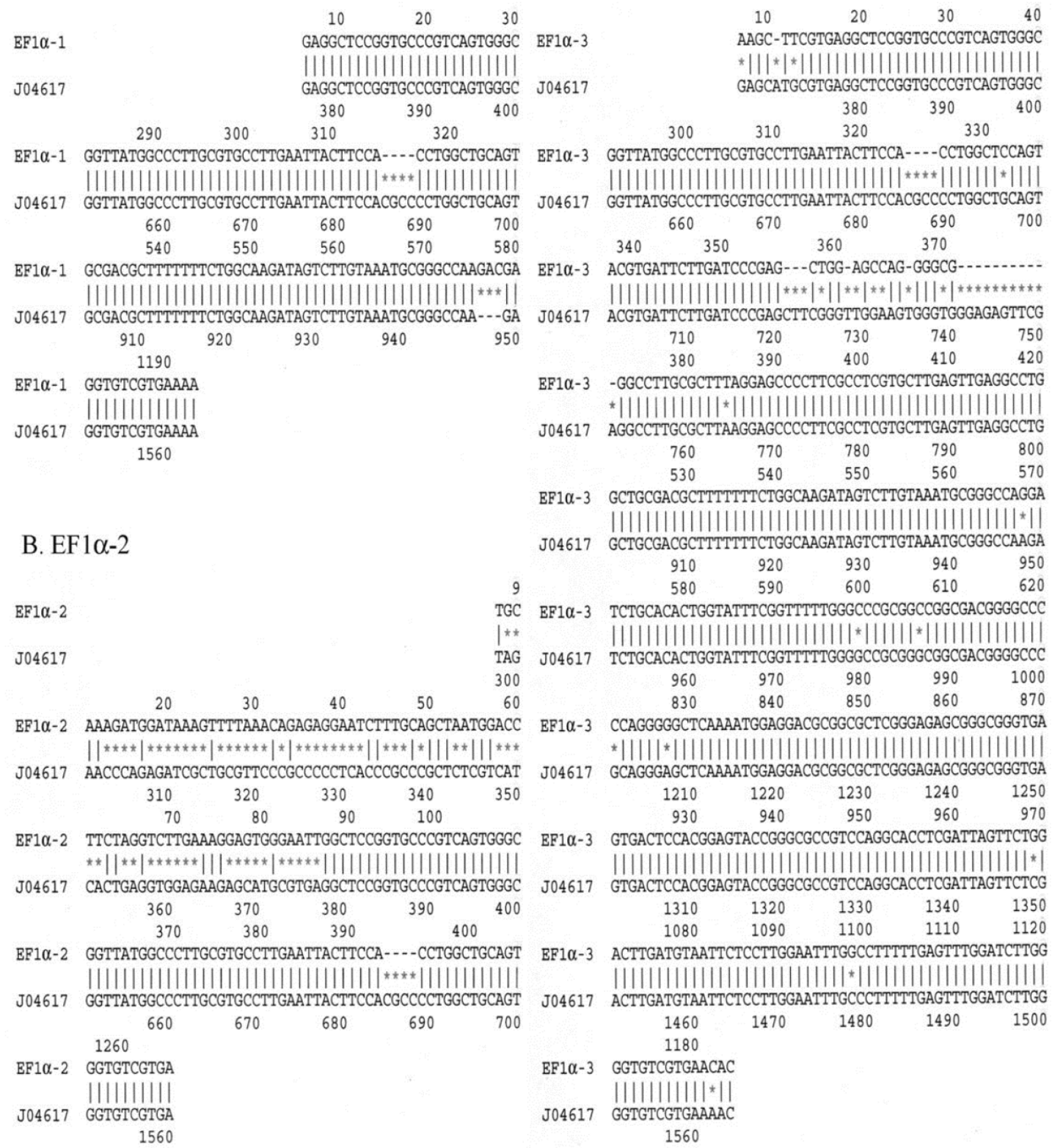

Figure 3. Comparison of the sequences from the $\mathrm{EFI} \alpha-\mathrm{I}, \mathrm{EFI} \alpha-2$ and $\mathrm{EFI} \alpha-3$ promoters with that of $046 \mathrm{I} / 7$ (the $\mathrm{EFI} \alpha$ sequence from GenBank; accession number in NCBI). A. EFI $\alpha-1$ promoter; B. EFI $\alpha-2$ promoter; C. EFI $\alpha-3$ promoter. Each “*” indicates a sequence difference from that of J046I7. 
Thus, the sequences of the EF1 $\alpha-1$ and EF1 $\alpha-2$ promoters are quite similar, except for the $81 \mathrm{bp} \mathrm{SV40}$ small antigen sequence at the 5 '-end of EF1 $\alpha-2$. However, this extra sequence apparently had little effect on driving transgene expression. Conversely, the sequence differences observed in the EF1 $1-3$ promoter, particularly the first and third ones described above, likely accounted for the considerably lower activity observed with this promoter. The EF1 $\alpha$ promoter should have broad activity in multiple cell types and therefore might be widely useful for applications in human gene transfer (6). All three of the promoters studied here are called EF1 $\alpha$ promoters in the literature, but they are not equivalent. Clearly, the use of the EF1 $\alpha-3$ promoter by investigators could result in dramatically lower transgene expression.

\section{Acknowledgements}

Our research is supported by the Division of Intramural Research, National Institute of Dental and Craniofacial Research, NIH.

\section{Competing Interests}

The authors have declared that no competing interest exists.

\section{References}

1. Benihoud K, Yeh P, Perricaudet M. Adenovirus vectors for gene delivery. Curr Opin Biotechnol. 1999; 10:440-447.

2. Orphanides G, Reinberg D. A unified theory of gene expression. Cell 2002; 108:439-451.

3. Linthout SV, Collen D, Geest BD. Effect of promoters and enhancers on expression, transgene DNA persistence, and hepatotoxicity after adenoviral gene transfer of human apolipoprotein A-I. Hum Gene Ther. 2002; 13:829-840.

4. Gambotto A, Dworacki G, Cicinnati V, et al. Immunogenicity of enhanced green fluorescent protein (EGFP) in BALB/c mice: identification of an H2-Kd-restricted CTL epitope. Gene Ther. 2000; 7:2036.

5. Oh JH, Sohn HY, Kim JM, et al. Construction of multi-purpose vectors, pCNS and pCNS-D2, are suitable for collection and functional study of large-scale cDNAs. Plasmid. 2004; 51:217.

6. Zheng C, Baum BJ. Evaluation of viral and mammalian promoters for use in gene delivery to salivary glands. Mol Ther. 2005; 12:528-536.

7. Mizushima S, Nagata S. pEF-BOS, a powerful mammalian expression vector. Nucleic Acids Res. 1990; 18:5322.

8. Brown AM, Rusnock EJ, Sciubba JJ, Baum BJ. Establishment and characterization of an epithelial cell line from the rat submandibular gland. J Oral Pathol Med. 1989; 18:206-213.

9. Yanagawa T, Hayashi Y, Nagamine S, et al. Generation of cells with phenotypes of both intercalated duct-type and myoethelial cells in human parotid gland adenocarcinoma clonal cells grown in athymic nude mice. Virchows Arch B Cell Pathol Incl Mol Pathol. 1986; 51:187-195.

10. Shirasuna K, Sato M, Miyasaki T. A neoplastic epithelial duct cell line established from an irradiated human salivary gland. Cancer 1981; 48:745-752.

11. Graham FL, Smiley J, Russel WC, Nairn R. Characteristics of a human cell lines transformed by DNA from human adenovirus type 5. J Gen Virol. 1977; 36:59-74.

12. Baker A, Saltik M, Lehrmann H, et al. Polyethylenimine (PEI) is a simple, inexpensive and effective reagent for condensing and linking plasmid DNA to adenovirus for gene delivery. Gene Ther. 1997; 4:773-782.

13. Meunier-Durmort C, Grimal H, Sachs LM, et al. Adenovirus enhancement of polyethylenimine-mediated transfer of regulated genes in differentiated cells. Gene Ther. 1997; 4:808-814.

14. Mastrangeli A, O'Connell B, Aladib $\mathrm{W}$, et al. Direct in vivo adenovirus-mediated gene transfer to salivary glands. Am J Physiol. 1994; 266(G):1146-1155.

15. Delporte $\mathrm{C}, \mathrm{O}^{\prime}$ Connell $\mathrm{BC}, \mathrm{He} \mathrm{X}$, et al. Increased fluid secretion after adenoviral-mediated transfer of the aquaporin- 1 cDNA to irradiated rat salivary glands. Proc. Natl. Acad. Sci. U S A. 1997; 94:3268-3273.
16. He X, Goldsmith CM, Marmary Y, et al. Systemic action of human growth hormone following adenovirus-mediated gene transfer to rat submandibular glands. Gene Ther. 1998; 5: 537-541.

17. Uetsuki T, Naito A, Nagata S, Kaziro Y. Isolation and characterization of the human chromosomal gene for polypeptide chain elongation factor-1 alpha. J Biol Chem. 1989; 264:5791-5798.

18. Messeguer X, Escudero R, Farre D, et al. PROMO: detection of known transcription regulatory elements using species-tailored searches. Bioinformatics 2002; 18:333-334.

19. Farre D, Roset R, Huerta $M$, et al. Identification of patterns in biological sequences at the ALGGEN server: PROMO and MALGEN. Nucleic Acids Res. 2003; 31:3651-3653.

20. Tsunoda T, Takagi T. Estimating transcription factor bindability on DNA. Bioinformatics. 1999; 15:622-630. 